\title{
Study on the Motor Control System based on Microcontroller
}

\author{
HUANG rui ${ }^{1, a}$ \\ ${ }^{1}$ Chongqing college of electronic engineering, Chongqing, 401331,China) \\ a2231066194@qq.com
}

Keywords: Motor Control System, Microcontroller, Electricity System

\begin{abstract}
With the continuous improvement of China's economic strength, science and technology to provide a strong guarantee in the future development of our country. In today's science and technology growing, electrical systems gradually become highly integrated, meanwhile, relevant science and technology has been constantly progress and breakthroughs. Among them, the rate of technological development mainly in the SCM system, led by particularly evident not only saves a great extent on the economic costs, but also to promote the development and popularization of the integrated circuit.
\end{abstract}

\section{Introduction}

Now, with electronic technology, control and changes in technology and the motor body, and the interface between the conventional motor classification increasingly blurred. On the conventional stepping motor, the stepping motor can be simply defined as: According to the input pulse signal, each time changing the magnetized state proceeds at an angle or length of the excitation state does not change its position to maintain a certain stationary motor. Broadly speaking, the stepper motor is a brushless DC motor controlled by electric pulses can also be seen as a synchronized speed and pulse frequency synchronous motor in a certain frequency range. Stepper motor has its own characteristics, summed up:

The digital signal can be used for direct open-loop control, the entire system is simple and inexpensive; Receives a digital signal directly, without having to digital to analog conversion, easy to use. The displacement of the input pulse signal corresponding to the number, the step error is not accumulated, can be composed of relatively simple structure but with a certain precision open-loop control system may also require more precision when the composition of the closed-loop control system; 4 brushless , motor body parts, high reliability; 5 . easy to start, stop, reversing and shifting, responsive right; 6 stops, can have self-locking function; 7 big step angle range of options available in Select several tens angle to 180 degree range. In the case of small steps, usually at the low speed high torque operation, which can directly drive the load without reducer work; 8 speed can be smoothly adjusted within a relatively wide range. At the same time using a stepper motor controller can control several they are completely synchronous operation; 9 can not directly use an ordinary AC power supply.

\section{The Concept of SCM Control System}

CPU as the core of the whole chip control system, which played a leading role in the whole system, by strengthening and upgrading the CPU, effectively promote the production control system are changing more convenient. Therefore, the single-chip control system throughout the process design is the most critical link in the link needs to be sophisticated design, but also need to choose the appropriate microcontroller and transmitter. In related parameter detection member is also required for modeling and design deployment, hardware and software system designed by the scientific and effective only after the completion of commissioning work can be carried out. In the design process the entire SCM system is sure to post the relevant hardware sufficient understanding of related software design, which can control the mathematical model of the object deeper inquiry and analysis.

It should target the specific circumstances of the accused full and comprehensive understanding of the overall design before making system, whether the controlled object has a full understanding 
of the overall design has a direct impact. Design of overall program design method has many kinds, which can select the type of control system according to the actual demand, the main types of closed-loop system in which the open-loop system. Among the control system, the accuracy of the detection element will SCM control system has a very important role, under normal circumstances should the measuring element make a reasonable choice to ensure the accuracy of the measured parameters. Actuator control system as a whole, one of the most important part, when it needs to consider not only the design of the control algorithms, but also should be combined with the actual demand.

\section{Overview of Motor Control System}

Machine control system is a complete organic whole, by the motion control system and operational control system. By the operating system to operation by the operator into motion control system capable of receiving electrical signals, along with the motion control system to respond and complete the operation. Motion control is a technology on how the object position and speed control. A typical motion control system shall be composed of three parts: the control part, driving part, the operative part.

Stepper motor is an electrical pulse into the angular displacement of the implementing agency. When the stepping driver receives a pulse signal, it will drive a stepper motor to set the direction of rotation of a fixed angle, its rotation is fixed angle step by step operation. Run stepper motor drives have an electronic device, such a device is a stepper motor drive, which is a pulse signal of the control system, to be enlarged in order to drive a stepper motor. Stepper motor speed proportional to the frequency of the pulse signal, the frequency of the control pulse signal can be accurately motor speed; control the number of step pulses, the motor can be precisely positioned. Thus a typical stepper motor drive control system mainly consists of three parts: a step controller driver (the controller output pulse is amplified to drive a stepper motor), the stepper motor. Different control schemes, stepper controllers, drives, there are different types. In the first chapter which has been introduced. The following highlights a microcontroller as the controller of the stepper motor control system.

Stepper motors are digitally controlled motor, its greatest feature is to be controlled by the input pulse signal, the total angle of rotation of the motor is determined by the number of input pulses, and the motor speed is determined by the pulse signal frequency. It has an input shaft rotation angle is proportional to the pulse motor characteristics and, converting the pulse signal into angular displacement, that is, to a pulse signal, the stepping motor is rotated at an angle, so it is suitable for single-chip control.

\section{SCM Software and Hardware Design}

SCM higher level of integration and it contains an internal memory and I / O control lines, however, extend an interface link is still need to focus on which wants to expand the structure of the microcontroller. There are two main programs. The first one is later refined interface board and the main structure of this system is the core program of the main system clock circuit, an address decoder, I / O interface design, input, display or the like. The second control system according to the needs, this method requires the use of a suitable extension to the chip design.

Software system is a whole system, the most important part, which has played a role in the effect of the actuator to a certain extent. In the single-chip control system, the software design is usually divided into two kinds, namely, software design and application software design. System software features for the entire process management SCM system, in the process of system software design software should ensure the stability of the system. In the design of the matching and unity it should be noted that software and hardware systems, system software are: management functions, DISPLAY, etc., as the system software to control the entire system, the core of the program for the single-chip control system plays a key role strengthen the design and development of software features can effectively reduce the hardware cost of the investment, and thus cost savings. 
In the course of the single-chip system design, to achieve the same general function, not only can be implemented using software, can also be implemented in hardware. Under normal circumstances, we will try to implement the functions of the application software to achieve the purpose of saving funds. If the system requires a strong real-time, you can achieve this functionality by hardware, for some relatively large amount of computation systems or more complex calculations, it can be applied to the high-level language programming. SCM system will generally devised a self-diagnostic program to facilitate all the time on the SCM system can perform diagnostic work. In certain high reliability requirements of the environment should be properly designed watchdog circuit and software traps, which can effectively prevent the occurrence of run error and other conditions. Application software have features are real-time, real-time system is a control system, so the execution speed of the software have specific requirements, which means that the software should be complete processing, computing, and other related operations within a certain period. During the program design process, as much as possible in order to save memory and enhanced adaptability. This requires reform process with common characteristics, so the choice of a modular structure in the process, should be as prepared by the common program subroutine come out.

\section{Commissioning SCM Control System}

The main purpose is to debug and troubleshooting can effectively solve the problems, which mainly contains the error process can be divided into two kinds of errors and failures of design. By design principles as the main basis for doing so enter the corresponding experimental prototype hardware debugging stage. Debug hardware circuit usually order: firstly should CPU clock circuit rational examination Secondly ABUS / DBUS of time-multiplexing capabilities for scientific testing, but also should I / O address of the adapter fault investigation. Finally, should the end-user I / O device for debugging.

The simulation plugs on the simulator to debug allowing you to see whether each circuit and reached the conditions required by the design. Due to the relatively strong empirical work belongs to the content of the work, so generally speaking, do you want to complete a one-time kind of mechanism is almost impossible, are the basic needs of the prototype debugging. More common debugging method: First the software can be programmed to debug, and then circuit design system one by one inspection and commissioning of the work, and finally for the MONITOR debug work. Software debugging method according to the actual situation of the device may be, the main debugging method were: cross-assembler debugging method, manual assembly and so on. After debugging through these several ways, so they can quickly enter the object code in the EPROM by the writer, so that the device can be a normal system operation. Software and hardware simulation debugging can effectively identify mismatches between software and hardware is present, and thus constituted by repeated debugging machine, then the next step of commissioning work.

\section{Conclusion}

Motor control system has a simple structure, reliable operation and easy maintenance, etc., across the range of applications, including industrial sector. As a single-chip processor motor control system greatly reduces the cost, but also greatly simplifies the wiring of the PCB, PCB layout makes it easier. SCM motor control system use software control, high speed, high precision and easy operation. With the development of manufacturing technology and mechatronics, automation equipment needs, requires motor control system with high efficiency, high precision and high reliability, so a single-chip motor control system has become an inevitable trend.

\section{References}

[1] Cheng Fangxiao, Xu Hongguo, Sunying Study on optimization for vehicle power train parameters [C]. The Ninth Internation Conference on Electronic Measurement \& Instruments 2009 (3): 989-992. 
[2] Gaosong, Gaoyan Study on power train parameters matching of series hybrid city bus [C]. Vehicular Electronics and Safety, 2006: 449-453.

[3] Xiong Weiwei, Shujie, Zhangyong Parameters matching for a series-parallel hybrid electric27 bus powertrain system [J]. Journal of Shanghai Jiaotong University, 2007, 42 (8): 1324-1328.

[4] HOSHI Nobukazu, ASAHI Norihiro Control Strategies for a Novel Inverter Which can Independently Control Two Permanent Magnet Synchronous Motors [J]. 13th European Conference on Power Electronics and Applications, 2009, 52 (1):79-88..

[5] Takano.W, Matsushita.A Recognition of Human Driving Behaviors based on Stochastic Symbolization of Time Series Signal [C]. IEEE / RSJ International Conference on Intelligent Robots and Systems 2008: 167-172.

[6] Yoshifumi.K, Koji.O. A Modeling Method for Predicting Driving Behavior Concerning with Driver's Past Movements [C]. IEEE International Conference on Vehicular Electronics and Safety Columbus 2008: 132-136.

[7] Aoude.G.S, Desaraju.V.R Behavior Classification Algorithms at Intersections and Validation Using Naturalistic Data [C]. IEEE Intelligent Vehicles Symposium, 2011. 601-606. 